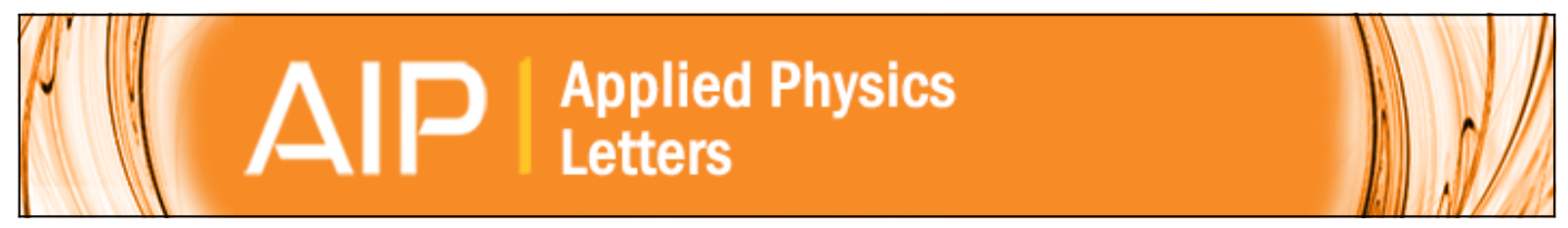

\title{
Electric-current-driven vortex-core reversal in soft magnetic nanodots
}

Sang-Koog Kim, Youn-Seok Choi, Ki-Suk Lee, Konstantin Y. Guslienko, and Dae-Eun Jeong

Citation: Applied Physics Letters 91, 082506 (2007); doi: 10.1063/1.2773748

View online: http://dx.doi.org/10.1063/1.2773748

View Table of Contents: http://scitation.aip.org/content/aip/journal/apl/91/8?ver=pdfcov

Published by the AIP Publishing

\section{Articles you may be interested in}

Radial-spin-wave-mode-assisted vortex-core magnetization reversals

Appl. Phys. Lett. 100, 172413 (2012); 10.1063/1.4705690

Out-of-plane current controlled switching of the fourfold degenerate state of a magnetic vortex in soft magnetic nanodots

Appl. Phys. Lett. 96, 072507 (2010); 10.1063/1.3310017

Understanding eigenfrequency shifts observed in vortex gyrotropic motions in a magnetic nanodot driven by spinpolarized out-of-plane dc current

Appl. Phys. Lett. 93, 182508 (2008); 10.1063/1.3012380

Reliable low-power control of ultrafast vortex-core switching with the selectivity in an array of vortex states by inplane circular-rotational magnetic fields and spin-polarized currents

Appl. Phys. Lett. 92, 022509 (2008); 10.1063/1.2807274

Effect of the classical ampere field in micromagnetic computations of spin polarized current-driven magnetization processes

J. Appl. Phys. 97, 10C713 (2005); 10.1063/1.1853291

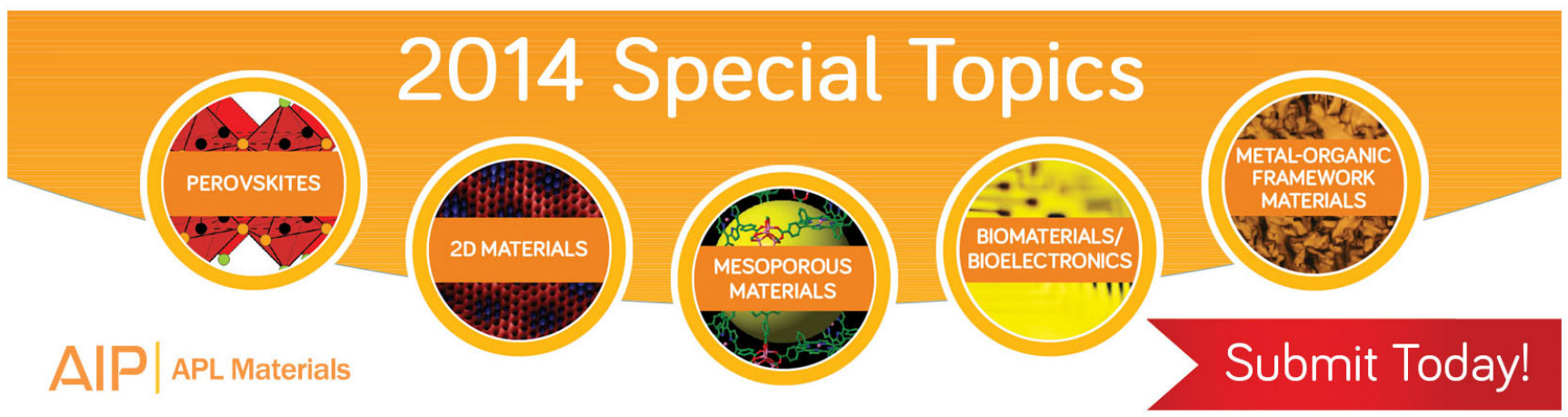




\title{
Electric-current-driven vortex-core reversal in soft magnetic nanodots
}

\author{
Sang-Koog Kim, ${ }^{\text {a) }}$ Youn-Seok Choi, Ki-Suk Lee, \\ Konstantin Y. Guslienko, and Dae-Eun Jeong \\ Research Center for Spin Dynamics \& Spin-Wave Devices and Nanospintronics Laboratory, \\ Department of Materials Science and Engineering, College of Engineering, Seoul National \\ University, Seoul 151-744, Republic of Korea
}

(Received 16 April 2007; accepted 30 July 2007; published online 22 August 2007)

\begin{abstract}
The authors report on electric-current-driven vortex-core (VC) reversal (switching) and the accompanying spin-wave emission, driven by spin-polarized ac currents of different amplitudes and frequencies, investigated by micromagnetic calculations of the dynamic evolution of a magnetic vortex in Permalloy nanodots. The magnetization orientation of the $\mathrm{VC}$ is effectively switchable between its upward and downward bistates and controllable by applying current above its threshold density, but with sufficiently small magnitude at frequencies close to the vortex eigenfrequency. This $\mathrm{VC}$ reversal phenomenon occurs through the creation of a vortex-antivortex pair and the subsequent annihilation of the initial vortex and the created antivortex, when the velocity of the initial VC reaches its critical value of approximately $340 \pm 20 \mathrm{~m} / \mathrm{s}$ for the given material and geometry. In the course of these serial processes and immediately after VC switching, strong spin waves are emitted. These results provide physical insights into how and when current-driven VC switching takes place, thereby offering a means to manipulate bistate VC orientations. (c) 2007 American Institute of Physics. [DOI: 10.1063/1.2773748]
\end{abstract}

Magnetic vortices (MVs) are observed typically in patterned or continuous soft magnetic thin films. The MV structure consists of in-plane curling magnetizations (Ms) and out-of-plane Ms at the core region. ${ }^{1-4}$ The vortex-core (VC) region ranges in size from 10 to $20 \mathrm{~nm} .^{2,3}$ This vortex state can be used as a memory unit cell in future memory devices, because not only does the MV have two discrete states of upand down-core orientations and two rotation senses (clockwise or counterclockwise) of the in-plane Ms but also the MV state is stable in sufficiently small lateral sizes $(>20 \mathrm{~nm})$ and thicknesses $(>2 \mathrm{~nm})$ of nanoelements. ${ }^{5}$ Also of recent and growing interest are the dynamic properties of a MV in response to driving forces such as magnetic fields and spin-polarized currents, owing to its nontrivial static ${ }^{1-6}$ and dynamic properties ${ }^{7-11}$ as well as promising potential applications to information storage devices. ${ }^{12,13}$

From a technological point of view, it is thus of great importance to readily control (switch) the VC orientation. However, such switching has been known to be possible only with the application of a strong static magnetic field, i.e, $H>2.5 \mathrm{kOe}$ along the $\mathrm{VC}$ orientation in order to overcome a huge energy barrier to the VC reversal. ${ }^{14}$ On the contrary, recently Van Waeyenberge et al. ${ }^{15}$ experimentally demonstrated, using a time-resolved scanning $\mathrm{x}$-ray microscope, that a VC in a Permalloy $\left(\mathrm{Ni}_{80} \mathrm{Fe}_{20}, \mathrm{Py}\right)$ square dot can be switched even with a small-amplitude oscillating magnetic field (field pulse). In addition, spin-polarized current has been used to manipulate the MV state in nanodots, ${ }^{16,17}$ and ac in-plane current was used to excite the VC eigenmodes. ${ }^{18}$ Moreover, electrical switching of VC orientation was promisingly demonstrated by both numerical calculation and experimental verification. ${ }^{19}$ These achievements would be sufficient to attract much attention, because not only the

a) Author to whom correspondence should be addressed; electronic mail: nonvolatile bistates of a MV with either up- or down-core orientation can be scaled down with its ultrahigh density but also the VC bistates can be readily manipulated by currents passing directly through the vortex structure. However, detailed understanding of current-driven VC switching is still lacking. In this letter, we report numerical calculations of the M dynamics of a MV state in a Py nanodot in response to alternating current of different amplitudes $I_{0}$ and frequencies $\nu$ around the resonant eigenfrequency $\nu_{0}$ of the dot. The nontrivial mechanism of $\mathrm{VC}$ reversal and its criteria related to the velocity of $\mathrm{VC}$ motion, as well as the additional phenomenon of spin-wave radiation immediately following the reversal, are elucidated.

In the present numerical simulations, we used the Landau-Lifshitz-Gilbert (LLG) simulator, ${ }^{20}$ which enables the calculation of $\mathbf{M}$ dynamics based on the LLG equation of motion with an additional term describing the torque exerted by spin-polarized current. ${ }^{21,22}$ This term was used in the form of $\mathbf{T}_{s}=-(\mathbf{u} \cdot \nabla) \mathbf{m}$, as suggested in Ref. 22, where $m=\mathbf{M} / M_{s}$, $\mathbf{u}=-\mathbf{j} P g \mu_{B} /\left(2 e M_{s}\right)$ with the current density $j$, the spin polarization $P$ of the current $(P=0.7$ for Py), the Landé splitting factor $g$, the electron charge $e$, the saturation magnetization $M_{s}$, and the Bohr magneton $\mu_{B} . \mathbf{T}_{s}$ corresponds to the "adiabatic" approximation, ${ }^{22}$ which for the case of $\mathrm{MV}$ dynamics was justified by Kasai et al. ${ }^{18}$ In the modeling, we chose a diameter of $2 R=600 \mathrm{~nm}$ and a thickness of $L=20 \mathrm{~nm}$ for the Py nanodisk. This model yields an initial M state of a single MV with the down-core orientation and counterclockwise in-plane $\mathbf{M}$ rotation, as shown in Fig. 1(a). The vortex gyrotropic eigenfrequency in the given model was determined to be $\nu_{0}=300 \mathrm{MHz}$. In-plane ac, $I(t)$ $=I_{0} \sin (2 \pi \nu t)$, of different $\nu$ and $I_{0}$ values were applied along the $x$ axis of the dot. Under the applied ac current, the VC position deviates from its initial center position and shows its spiral motion around the dot center, and then the VC orientation can be switched opposite to the initial 
(a)

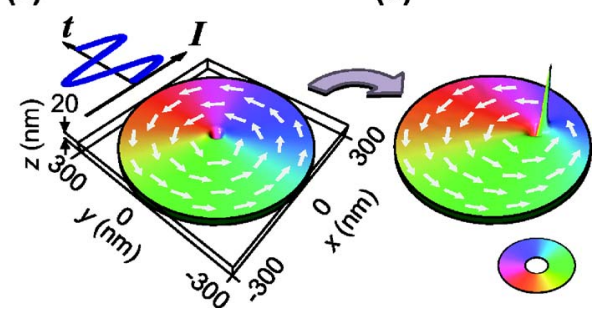

FIG. 1. (Color online) Geometry and dimension of the model Py nanodot. The in-plane ac is applied along the $x$ axis to the dot. The initial vortex state of the downward core orientation located in the middle of the disk and with counterclockwise in-plane $\mathbf{M}$ orientation in (a) is switched to the upward core orientation with the same in-plane $\mathbf{M}$ rotation in (b). The colors display the local $\mathbf{M}$, as indicated by the color wheel. The height in the images represents the out-of-plane component of the local Ms.

core orientation under some conditions, as shown in Fig. 1(b).

Figure 2(a) shows the resultant dynamic evolution of the VC motion at $\left(I_{0}, \nu\right)=(7.2 \mathrm{~mA}, 300 \mathrm{MHz})$, illustrated by perspective-view images of the out-of-plane $\mathbf{M}$ components (represented by height and color) at the indicated times. The applied values of $\nu$ and $I_{0}$ correspond to the $\nu_{0}$ and a current density $j_{0}=6 \times 10^{7} \mathrm{~A} / \mathrm{cm}^{2}$, respectively. Upon the application of $I(t)$, the $\mathrm{VC}$ begins to move and deviate in position from the dot center, and then shows its spiral-like motion, as indicated by its VC trajectory (blue curve with arrows), in the time interval of $t=0-16 \mathrm{~ns}$, shown in the top of Fig. 2(b). When the VC velocity reaches a critical value

(a)
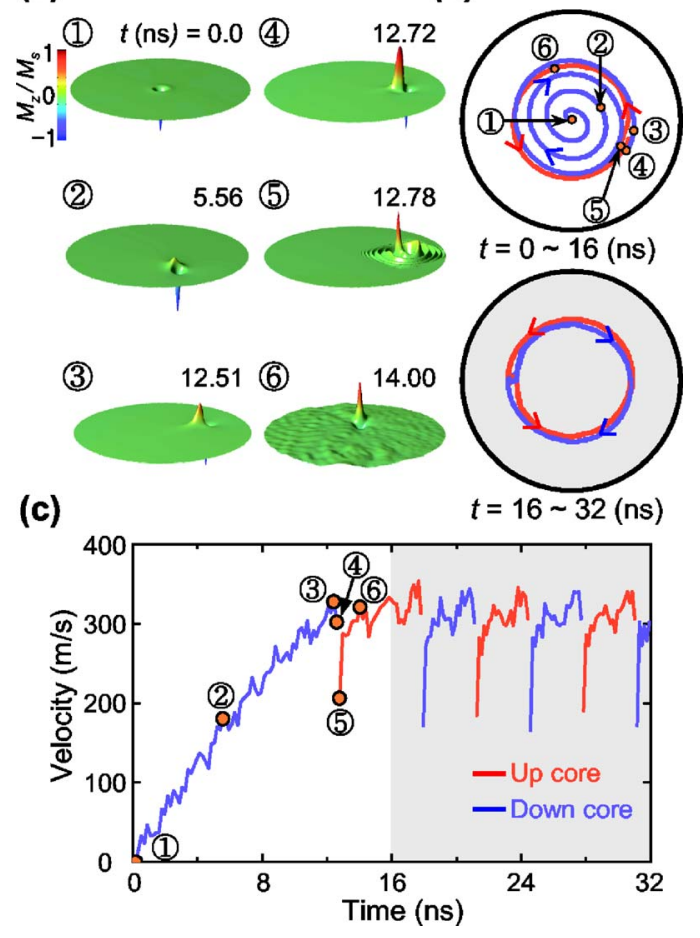

FIG. 2. (Color online) (a) Series of snapshot images illustrating the dynamic evolution of the $\mathrm{VC}$ gyrotropic motion and its reversal process, and the resultant spin-wave emission, driven by an ac of $I_{0}=7.2 \mathrm{~mA}$ (current density $j_{0}=6 \times 10^{7} \mathrm{~A} / \mathrm{cm}^{2}$ ) and $\nu=300 \mathrm{MHz}$. (b) The orbit trajectories of the VC gyromotions. (c) The velocity of the $\mathrm{VC}$ moving along the trajectories as a function of time. The blue and red colors indicate the initial VC and the reversed $\mathrm{VC}$, respectively. The numbers noted on the trajectory curve and velocity vs time curve, indicating each stage of the dynamic evolution, are the same as those shown in (a).

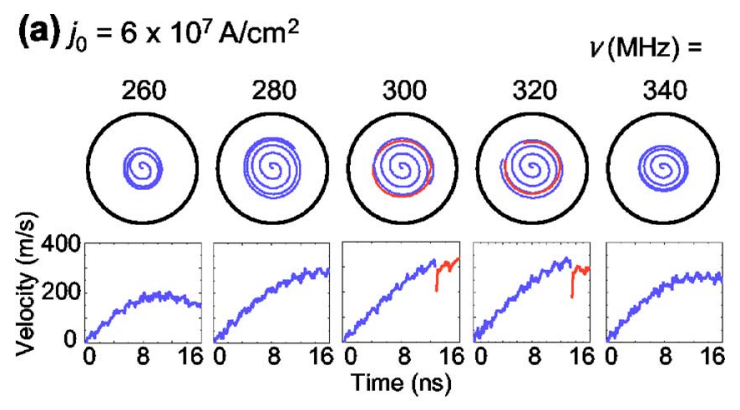

(b) $v=300 \mathrm{MHz}$

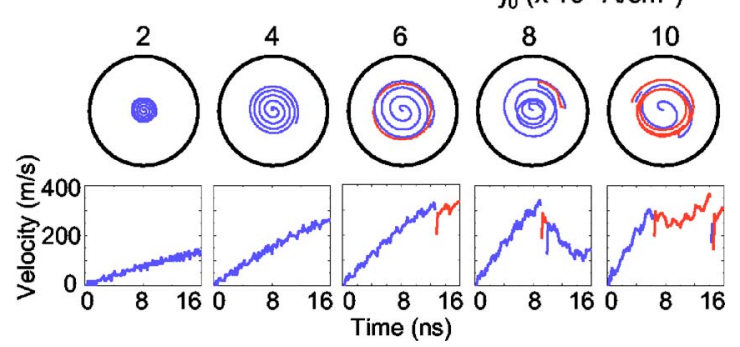

FIG. 3. (Color online) VC trajectories before the VC reversal or both before and after, and the velocities of the $\mathrm{VC}$ gyromotions for the various current parameters as indicated. (a) For $j_{0}=6 \times 10^{7} \mathrm{~A} / \mathrm{cm}^{2}, \nu$ varies from 260 to $340 \mathrm{MHz}$ in increments of $20 \mathrm{MHz}$. (b) For $\nu=\nu_{0}=300 \mathrm{MHz}, j_{0}$ varies from $2 \times 10^{7}$ to $1 \times 10^{8} \mathrm{~A} / \mathrm{cm}^{2}$ in increments of $2 \times 10^{7} \mathrm{~A} / \mathrm{cm}^{2}$. The blue and red colors indicate the initial $\mathrm{VC}$ before its reversal and the reversed VC after, respectively.

$v_{c}=340 \pm 20 \mathrm{~m} / \mathrm{s}$ in the given dot, the initial down-core (blue curve) orientation is switched to the up-core (red) orientation (see moment 5). The reversed VC changes the sense of its gyrotropic motion from the clockwise (blue) to counterclockwise (red) rotation. ${ }^{7,8}$ For $\nu=\nu_{0}$, it is clear that VC reversal repeatedly takes place when the reversed VC velocity reaches $v_{c}=340 \pm 20 \mathrm{~m} / \mathrm{s}$ again, as seen in Fig. 2(c). The first $\mathrm{VC}$ reversal occurs in a time of $12.75 \mathrm{~ns}$, and the next serial reversals occur every $3.3 \pm 0.15 \mathrm{~ns}$.

In order to investigate the effects of $\nu$ and $j_{0}$ on $\mathrm{VC}$ reversal, we conducted the same simulations with different $j_{0}$ and $\nu$ values. The $\mathrm{VC}$ trajectories in the time period of $t=0-16 \mathrm{~ns}$ and the VC velocity versus time are shown for different $\nu$ values at $j_{0}=6 \times 10^{7} \mathrm{~A} / \mathrm{cm}^{2}$ in Fig. 3(a) and for different $j_{0}$ values at $\nu=\nu_{0}=300 \mathrm{MHz}$ in Fig. 3(b). The results showed that the $\mathrm{VC}$ reversal can be effectively manipulated at $\nu$ close to $\nu_{0}$, and even at $\nu=\nu_{0}$ a sufficiently large current density is required for the VC switching. For example, at $\nu=\nu_{0}$, current densities less than $4 \times 10^{7} \mathrm{~A} / \mathrm{cm}^{2}$ do not lead to VC reversal, but affecting the orbital size of the $\mathrm{VC}$ gyromotions. According to the velocity-versus-time curves shown in Fig. 3, VC reversal always occurs when the VC velocity reaches $v_{c}=340( \pm 20) \mathrm{m} / \mathrm{s}$ in the given dot. It is obvious that the VC orientation switches very effectively in the cases of $\nu \sim \nu_{0},{ }^{11}$ and that at the given value of $\nu$, there exists a certain threshold value of $j_{0}$ for $\mathrm{VC}$ reversal. Driving force parameters such as $\nu$ and $j_{0}$ do not affect the value of $v_{c}$ that is given for the material and geometry. In order to identify the parameters governing $v_{c}$, further quantitative studies employing both micromagnetic simulations and theoretical approaches are necessary.

As the next step, it is necessary to clarify the underlying mechanism of the observed VC switching. We plotted the detailed dynamic $\mathbf{M}$ distributions in a local area nearby the initial VC position at the times indicated in Fig. 4. A close 


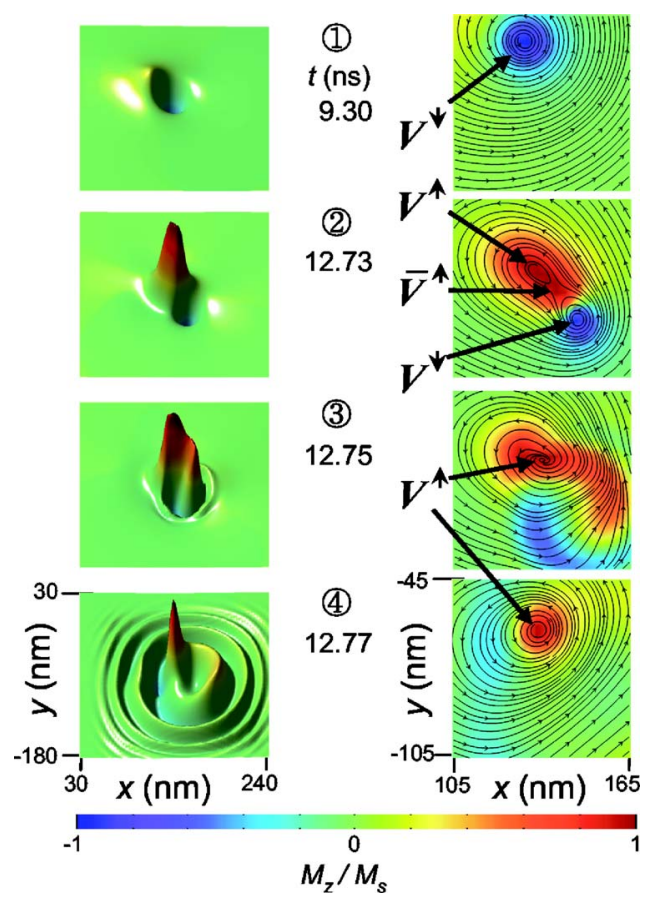

FIG. 4. (Color online) Snapshot images of the local M distribution taken at the indicated times from the perspective (left column) and plane (right column) views. The color and height of the surfaces in the left column indicate the local out-of-plane $\mathbf{M}$ normalized by the saturation value, $M_{z} / M_{s}$. The streamlines with the small arrows in the right column indicate the in-plane direction of the local M. The symbols $V^{\uparrow, \downarrow}$ and $\mathbf{V}^{\uparrow, \downarrow}$ represent $\mathrm{V}$ and $\mathrm{AV}$, respectively. Their superscripts indicate the "up" $(\uparrow)$ and "down" $(\downarrow)$ core orientations.

look at the microstructure of each stage of the dynamic evolution shows that the VC reversal process is more complicated than a simple switching between the down- and upcore orientations. With increasing $\mathrm{VC}$ velocity, the initial MV structure begins to deform, the VC deformation maximizes, and the in-plane curling $\mathbf{M}$ structure becomes more elongated (indicated in moment 2). These deformations lead to the concentration of the dipolar and exchange energies, and hence their energy densities markedly increase in the area nearby the core position. The out-of-plane $\mathbf{M}$ component in this area also increases at the expense of the dipolar energy as the in-plane $\mathbf{M}$ structure of the MV becomes more deformed. Thus, a vortex-antivortex (V-AV) pair $\left(\mathrm{V}^{\uparrow}-\overline{\mathrm{V}}^{\uparrow}\right)$ eventually nucleates nearby the initial VC (at moment 2 ). The nucleated V-AV pair has parallel core orientations opposite to that of the original VC $\left(\mathrm{V}^{\downarrow}\right)$. Due to their attractive interaction, accordingly, the nucleated $\overline{\mathrm{V}}^{\uparrow}$ and the original $\mathrm{V}^{\downarrow}$ move closer, ${ }^{23}$ and finally annihilate (see moment 3 ), reducing the exchange energy that was previously stored as a result of the presence of the additional VCs in the $\mathrm{V}^{\uparrow}-\overline{\mathrm{V}}^{\uparrow}$ pair. Therefore, the MV state in the dot after the reversal process is the remaining $\mathrm{V}^{\uparrow}$ (see moment 4). These processes occur continuously and repeatedly in response to the ac, as already shown in Fig. 2. Immediately after V-AV annihilation, the excess energy offered by the ac is emitted in the form of spin waves (see moment 4). ${ }^{23}$ The spin waves, of discrete gigahertz-range frequencies, are emitted from the V-AV annihilation point, then propagate to the dot border and reflect from it, forming an interference pattern. ${ }^{24}$ We found that this unexpected dynamic process is mediated by the serial process of the creation of a V-AV pair and the subsequent annihilation of the initial MV and the created AV. Such a reversal mechanism has also been found in VC reversals driven by small-amplitude in-plane oscillating magnetic fields. ${ }^{15,23-25}$ Current- and magnetic-field-driven VC reversals show their similarity in the underlying mechanism and are pure dynamic ultrafast $(<0.1 \mathrm{~ns})$ processes [see the time interval from state 3-5 in Fig. 2(c)], reflecting the relation of the time scale to the strong exchange interaction. This allows the VC reversal to occur on a few tens of picosecond scale with relatively low currents of $6-10 \mathrm{~mA}$ in the given dot when $\nu \sim \nu_{0}$. Further studies on VC reversal criteria are underway.

To conclude, the results of the present study amount to a signal opportunity to use VCs for ultrafast information storage and recording in the subterahertz frequency range and as a source of high-amplitude spin waves.

This work was supported by Creative Research Initiatives (ReC-SDSW) of MOST/KOSEF.

${ }^{1}$ A. Hubert and R. Schafer, Magnetic domains (Springer, Berlin, 1998).

${ }^{2}$ T. Shinjo, T. Okuno, R. Hassdorf, K. Shigeto, and T. Ono, Science 289, 930 (2000).

${ }^{3}$ A. Wachowiak, J. Wiebe, M. Bode, O. Pietzsch, M. Morgenstern, and R. Wiesendanger, Science 298, 577 (2002).

${ }^{4}$ S.-K. Kim, K.-S. Lee, B.-W. Kang, K.-J. Lee, and J. B. Kortright, Appl. Phys. Lett. 86, 052504 (2005); S.-K. Kim, J. B. Kortright, and S.-C. Shin, ibid. 78, 2742 (2001).

${ }^{5}$ K. L. Metlov and K. Y. Guslienko, J. Magn. Magn. Mater. 242-245, 1015 (2002).

${ }^{6}$ R. P. Cowburn, D. K. Koltsov, A. O. Adeyeye, M. E. Welland, and D. M. Tricker, Phys. Rev. Lett. 83, 1042 (1999).

${ }^{7}$ K. Y. Guslienko, B. A. Ivanov, V. Novosad, Y. Otani, H. Shima, and K. Fukamichi, J. Appl. Phys. 91, 8037 (2002).

${ }^{8}$ S. B. Choe, Y. Acremann, A. Scholl, A. Bauer, A. Doran, J. Stohr, and H. A. Padmore, Science 304, 420 (2004).

${ }^{9}$ J. P. Park, P. Eames, D. M. Engebretson, J. Berezovsky, and P. A. Crowell, Phys. Rev. B 67, 020403(R) (2003).

${ }^{10}$ V. Novosad, F. Y. Fradin, P. E. Roy, K. S. Buchanan, K. Y. Guslienko, and S. D. Bader, Phys. Rev. B 72, 024455 (2005).

${ }^{11}$ K.-S Lee, K. Y. Guslienko, J.-Y. Lee, and S. -K. Kim, e-print arXiv:condmat/0703538.

${ }^{12}$ S. D. Bader, Rev. Mod. Phys. 78, 1 (2006).

${ }^{13}$ K. Bussmann, G. A. Prinz, S.-F. Cheng, and D. Wang, Appl. Phys. Lett. 75, 2476 (1999).

${ }^{14}$ N. Kikuchi, S. Okamoto, O. Kitakami, Y. Shimada, S. G. Kim, Y. Otani, and K. Fukamichi, J. Appl. Phys. 90, 6548 (2001).

${ }^{15}$ B. Van Waeyenberge, A. Puzic, H. Stoll, K. W. Chou, T. Tyliszczak, R. Hertel, M. Fähnle, H. Brückl, K. Rott, G. Reiss, I. Neudecker, D. Weiss, C. H. Back, and G. Schütz, Nature (London) 444, 461 (2006).

${ }^{16}$ T. Ishida, T. Kimura, and Y. Otani, Phys. Rev. B 74, 014424 (2006).

${ }^{17}$ J. Shibata, Y. Nakatani, G. Tatara, H. Kohno, and Y. Otani, Phys. Rev. B 73, 020403 (2006); J. Magn. Magn. Mater. 310, 2041 (2007).

${ }^{18}$ S. Kasai, Y. Nakatani, K. Kobayshi, H. Kohno, and T. Ono, Phys. Rev. Lett. 97, 107204 (2006); J. Magn. Magn. Mater. 310, 2351 (2007).

${ }^{19}$ K. Yamada, S. Kasai, Y. Nakatani, K. Kobayashi, H. Kohno, A. Thiaville, and T. Ono, Nat. Mater. 6, 269 (2007).

${ }^{20}$ For details, see http://llgmicro.home.mindspring.com/1, 1997.

${ }^{21}$ L. Berger, Phys. Rev. B 54, 9353 (1996); J. C. Slonczewski, J. Magn. Magn. Mater. 159, L1 (1996).

${ }^{22}$ S. Zhang and Z. Li, Phys. Rev. Lett. 93, 127204 (2004); A. Thiaville, Y. Nakatani, J. Miltat, and Y. Suzuki, Europhys. Lett. 69, 990 (2005).

${ }^{23}$ K.-S. Lee, S. Choi, and S.-K. Kim, Appl. Phys. Lett. 87, 192502 (2005).

${ }^{24}$ S. Choi, K.-S. Lee, K. Y. Guslienko, and S.-K. Kim, Phys. Rev. Lett. 98, 087205 (2007).

${ }^{25}$ R. Hertel, S. Gliga, M. Fähnle, and C. M. Schneider, Phys. Rev. Lett. 98, 117201 (2007). 